\title{
Efeito da enrofloxacina sobre a interação blastocisto endométrio e seu reflexo no desenvolvimento placentário e fetal em ratas
}

\author{
[Effect of enrofloxacin on the blastocyst endometrial interactions and their impact on \\ placental and fetal development in rats]
}

\author{
I.M.F. Melo, W.E. Silva, A.A.C. Teixeira*, V. Wanderley-Teixeira, S.B.F.P. Barreto, \\ C.J.C. Lapa Neto, F.M. Andrade
}

Universidade Federal Rural de Pernambuco - Recife, PE

\begin{abstract}
RESUMO
Estudos têm mostrado efeitos tóxicos da enrofloxacina em diversos tecidos. Assim, testou-se a hipótese de que a enrofloxacina pode interferir no desenvolvimento placentário e gerar efeitos adversos ao feto. A enrofloxacina $\left(\right.$ Baytril $^{\circledR}$ ) foi administrada em ratas, na dose de $5 \mathrm{mg} / \mathrm{kg}$, diariamente, IM, durante toda a gestação. As placentas foram analisadas morfologicamente, morfometricamente e imuno-histoquimicamente aos sete, 14 e 21 dias de prenhez. Os resultados mostraram que a enrofloxacina reduziu o número de sítios de implantação, o peso e a área total do disco placentário aos 14 e 21 dias de desenvolvimento, além dos elementos constituintes da placenta. A análise histoquímica não revelou alterações significativas no teor de fibras colágenas, elásticas e reticulares. O teste de Tunel mostrou atividade apoptótica apenas nas placentas com 14 dias de desenvolvimento de ambos os grupos, sendo mais intensa no grupo tratado. Não foi observado nenhum indício de malformação na cabeça, no tronco e nos membros dos neonatos. No entanto, houve uma redução significativa no número e no peso dos neonatos no grupo tratado, porém sem afetar o seu comprimento. Assim, concluiu-se que a enrofloxacina administrada na dosagem de $5 \mathrm{mg} / \mathrm{kg}$ durante prenhez em ratas interfere no número de embriões implantados e no desenvolvimento placentário. Isto sugere cautela na administração da enrofloxacina durante a gestação, pois a exposição contínua a esse antibiótico pode ter reflexos na redução do número e do peso da prole.
\end{abstract}

Palavras-chave: enrofloxacina, prenhez, placenta, imuno-histoquímica, reprodução

\begin{abstract}
Some studies have shown the toxic effects of enrofloxacin in various tissues. Thus, the hypothesis that enrofloxacin could interfere with placental development and generate adverse effects to the fetus was tested in this study. Enrofloxacin (Baytril $\left.{ }^{\circledR}\right)$ was administered in the dose of $5 \mathrm{mg} / \mathrm{kg}$ daily, i.m., throughout gestation in rats. The placentas were analyzed morphologically, morphometrically, and immunohistochemically on the 7,14 , and $20^{\text {th }}$ days of pregnancy. The results showed that enrofloxacin reduced the number of implantation sites, weight, and placental disk total area at 14 and 20 days of development, in addition to the element components of the placenta. The histochemical analysis did not reveal significant changes in the content of collagen, reticular, and elastic fibers. The TUNEL test showed apoptosis only in placenta development at 14 days in both groups and more intense in the treated group. Head, trunk, and limb malformations were not observed in the neonates. A significant reduction in the number and weight of neonates were observed in the treated group, however, without affecting their length. Thus, it was concluded that the administration of enrofloxacin, at the dosage of $5 \mathrm{mg} / \mathrm{kg}$ during pregnancy in rats, interferes in the number of implanted embryos and placental development. This suggests caution in the administration of enrofloxacin during pregnancy because continuous exposure to this antibiotic may have adverse effects, reducing the number and weight of the offspring.
\end{abstract}

Keywords: enrofloxacin, pregnancy, placental development, immunohistochemistry, reproduction

Recebido em 13 de abril de 2012

Aceito em 19 de fevereiro de 2014

*Autor para correspondência (corresponding author)

E-mail: alvaro@dmfa.ufrpe.br 


\section{INTRODUÇÃO}

Fluoroquinolonas são agentes antibacterianos que têm um amplo espectro de atividades contra bactérias Gram-positivas e Gram-negativas, utilizados tanto na medicina humana, como na veterinária (Abd-Allah et al., 2000). Apesar de esses agentes serem geralmente bem tolerados, efeitos adversos têm sido relatados, tais como: vômito, diarreia, tonturas, dor de cabeça, insônia e fototoxicidade (Owens e Ambrose, 2005). Somando-se a esses sintomas, algumas fluoroquinolonas, como ciprofloxacina, pefloxacina, DW-16 e moxifloxacina, podem causar danos tanto na matriz de tecidos conjuntivos como das cartilagens e dos tendões (Van der Linden et al., 2003), além de cardiotoxicidade e alterações no sistema nervoso central (Stahlmann, 2002; Kim et al., 2000, 2003). Esses efeitos colaterais resultaram na restrição dessas fluoroquinolonas em crianças e mulheres grávidas (Shehata e Nelson-Piercy, 2001).

A enrofloxacina (1-ciclopropil-7-(etil-1piperazinil)-6-fluoro-1, 4dihydro-4-oxo-3- ácido quinolonacarboxílico) é uma fluoroquinolona, desenvolvida apenas para uso em animais (Elmas et al., 2001), indicada para tratamento de infecções respiratórias, gastrointestinais, urinárias, piodermatites, otites, entre outros processos infecciosos (Marín et al., 2007). Parte da atividade antimicrobiana da enrofloxacina é atribuída ao seu metabólito principal, a ciprofloxacina (Otero et al., 2001).

Alguns estudos têm mostrado efeitos tóxicos da enrofloxacina em diversos tecidos. Gurbay et al. (2006) e Lim et al. (2008) relataram inibição da proliferação celular, indução da apoptose e fragmentação do DNA em células do tendão e condrócitos em cães e cavalos. Minta et al. (2005) evidenciaram in vitro citotoxicidade da enrofloxacina em células do mesencéfalo em ratos. Esse efeito tóxico da enrofloxacina, semelhantemente às outras fluoroquinolonas, pode ser devido à tendência de ela se acumular como resíduo em vários tecidos e órgãos, tais como: músculo, intestino, nódulos linfáticos, fígado, rins e útero, o que, muitas vezes, provoca toxicidade (Lemus et al., 2008).

$\mathrm{Na}$ reprodução, Aral et al. (2008), ao administrarem enrofloxacina, na dosagem de
$150 \mathrm{mg} / \mathrm{kg} /$ dia durante 15 dias, em ratos machos, observaram redução da quantidade e da mobilidade dos espermatozoides, anormalidades morfológicas no epidídimo, além de danos estruturais no tecido dos testículos. Lemus et al. (2009) demonstraram ainda que a enrofloxacina e a ciprofloxacina podem provocar a mortalidade de embriões de aves, pelo fato de interferirem no teor de vitelo e no desenvolvimento do saco vitelino. No entanto, não há relatos da ação desse fármaco sobre desenvolvimento da toxicidade quando administrado durante a prenhez em mamíferos, principalmente em relação à formação da placenta.

Crescimento e desenvolvimento da placenta são cruciais para o feto, e uma alteração nesses processos pode estar intimamente relacionada com a deficiência no desenvolvimento fetal (Katayama et al., 2002). Em camundongos e ratos, várias substâncias tóxicas podem induzir o desenvolvimento excessivo da apoptose placentária e prejudicar o crescimento fetal (Thota et al., 2005). Assim, testou-se a hipótese de que a enrofloxacina, administrada durante o período gestacional, pode interferir no desenvolvimento placentário. Dessa forma, avaliaram-se histologicamente, morfometricamente e imuno-histoquimicamente os sítios de implantação aos sete dias e as placentas aos 14 e 21 dias de prenhez.

\section{MATERIAL E MÉTODOS}

Foram utilizadas 40 ratas albinas (Rattus norvergicus albinus) da linhagem Wistar, com 90 dias de idade, procedentes do Biotério do Departamento de Morfologia e Fisiologia Animal, da Universidade Federal Rural de Pernambuco. O protocolo experimental foi aprovado pela Comissão de Ética institucional de número: 23082.010602/2010. As fêmeas que apresentaram três ciclos estrais regulares foram acasaladas e distribuídas ao acaso, em dois grupos: G I - ratas prenhes sem tratamento, e G II - ratas prenhes tratadas com enrofloxacina. Utilizou-se o Baytril $^{\circledR} \quad$ (enrofloxacina), na dosagem de $5,0 \mathrm{mg} / \mathrm{kg}$ a cada 24 horas, via intramuscular, por um período de sete, 14 e 21 dias de prenhez. O grupo I recebeu solução fisiológica na mesma dosagem por via intramuscular, por um período de sete, 14 e 21 dias de prenhez (Elmas et al., 2007). 
Cinco fêmeas de cada grupo foram anestesiadas no sétimo, $14^{\mathrm{O}}$ e $21^{\mathrm{o}}$ dias de prenhez com hidrocloridrato de cetamina $(80 \mathrm{mg} / \mathrm{kg})$ e xilazina $(6 \mathrm{mg} / \mathrm{kg})$, IM (Andrade, 2002), para retirada dos cornos uterinos contendo sítios de implantação e placentas, os quais foram fixados em formol a $10 \%$ e incluídos em parafina. Os sítios de implantação foram contados, e as placentas pesadas. Os cortes foram corados pela hematoxilina-eosina (H. E.), tricrômico de Mallory (fibras colágenas), orceína nítrica (fibras elásticas), impregnação argêntica (fibras reticulares) e analisados em microscópio de luz, da marca Olympus ${ }^{\circledR}$ BX49. A morfometria foi realizada pela captura de imagens por meio de câmera de vídeo Sony ${ }^{\circledR}$, acoplada ao microscópio Olympus $^{\circledR}$ BX50, e essas imagens foram submetidas ao aplicativo morfometria de linhas, calibrado em micrômetros, associado ao programa ImagemLab 2000 para Windows. Foram utilizadas de cada grupo cinco lâminas de placenta com 14 e 21 dias. A análise dos elementos do disco placentário foi realizada segundo a metodologia de Mayhew (2006). Em cada lâmina foram contados 10 campos aleatórios, com quatro repetições, perfazendo um total de 1000 pontos por lâmina, sendo quantificados os seguintes parâmetros: na região do labirinto: 1-trofoblasto sincicial, 2- vasos fetais e 3- vasos maternos; na região do trofospongio/células trofoblásticas gigantes: 1células trofoblásticas, 2- trofoblasto sincicial e 3células trofoblásticas gigantes. Para a detecção de apoptose, cinco lâminas silanizadas de cada grupo, as quais continham cortes de sítio de implantação e placentas com 14 e 21 dias de desenvolvimento, foram submetidas ao teste de Tunel.

As cinco fêmeas restantes de cada grupo foram acompanhadas durante toda a gestação até o nascimento dos filhotes, os quais foram contados, pesados em balança analítica, medidos com o auxílio de um paquímetro e analisados macroscopicamente para observação de alguma malformação visível. Os dados da quantificação dos sítios de implantações, o número de neonatos e seus respectivos pesos e tamanhos, além das mensurações do disco placentário, foram submetidos ao teste não paramétrico de KruskalWallis, e as médias foram comparadas pelo teste de Wilcoxon-Mann-Whitney $(\mathrm{p}<0,05)$.

\section{RESULTADOS}

A análise estatística do número de sítios de implantação nos animais dos grupos experimentais revelou que o tratamento com a enrofloxacina reduziu significativamente os sítios em relação ao controle. Houve uma redução estatisticamente significativa no peso das placentas das ratas tratadas com enrofloxacina, tanto aos 14 como aos 21 dias de desenvolvimento, quando se comparou ao peso dos animais do grupo controle (Tab. 1).

Os sítios de implantação nas ratas do grupo controle apresentaram-se totalmente inseridos na parede do útero (Fig. 1A). Histologicamente esses sítios mostraram-se constituídos por trofoblastos, alguns com atividade mitótica, citotrofoblastos poliploides e rica vascularização. O epitélio luminal no grupo controle apresentouse característico do tipo simples colunar, e na decídua foram visualizadas várias glândulas endometriais. Os sítios de implantação no grupo tratado apresentaram as mesmas características histológicas do controle (Fig. 1B).

A análise morfológica das placentas, com 14 e 21 dias de desenvolvimento, dos grupos experimentais não mostrou alterações histológicas significativas. Foi observada a região da decídua bastante vascularizada e a região do disco placentário bem desenvolvido, com as três camadas: a camada do labirinto, região mais externa e a mais espessa, caracterizada pela presença de numerosas lacunas contendo vasos maternos e fetais; a camada do trofospongio, na qual se observam trofoblastos indiferenciados; e a última camada, formada pelas células trofoblásticas gigantes, as quais se misturam com a decídua (Fig. 1C, 1D, $1 \mathrm{E}$ e $1 \mathrm{~F})$. A análise histoquímica não revelou alterações significativas no teor de fibras colágenas, elásticas e reticulares (Tab. 2). 
Efeito da enrofloxacina...

Tabela 1. Médias e desvio-padrão do número de sítios de implantação (7 dias) e peso(g) das placentas aos 14 e 21 dias de prenhez das ratas dos grupos experimentais

\begin{tabular}{cccc} 
Dias & GI & GII & $\mathrm{F}^{\mathrm{P}}$ \\
\hline 7 & $13,60 \pm 2,07 \mathrm{a}$ & $10,40 \pm 1,51 \mathrm{~b}$ & $2,182^{0,0159}$ \\
14 & $0,301 \pm 0,018 \mathrm{a}$ & $0,216 \pm 0,059 \mathrm{~b}$ & $1,430^{0,0079}$ \\
21 & $0,650 \pm 0,070 \mathrm{a}$ & $0,503 \pm 0,044 \mathrm{~b}$ & $2,765^{0,0111}$
\end{tabular}

*Médias seguidas pela mesma letra não diferem significativamente entre si pelo teste de Wilcoxon-Mann-Whitney $(\mathrm{P}<0,05)$.

Tabela 2. Histoquímica do sítio de implantação e disco placentário (14 e 20 dias) nos grupos experimentais. Reação intensa $(++)$, moderada $( \pm)$ e fraca $(+)$

\begin{tabular}{ccc}
\hline & GI & GII \\
\hline Fibras & & \\
colágenas & ++ & ++ \\
Fibras & & \\
elásticas & + & + \\
Fibras & & \\
reticulares & \pm & \pm \\
\hline
\end{tabular}

A análise estatística das médias da área total do disco placentário mostrou que os animais do grupo II apresentaram as menores médias, tanto aos 14 como aos 21 dias de prenhez, o que difere significativamente do grupo controle (Tab. 3). No grupo II, também foram evidenciadas diferenças significativas na região do labirinto para os parâmetros trofoblastos sinciciais e vascularização materna e fetal (Tab. 4). Na camada de trofospongio, a média das células trofoblásticas e dos trofoblastos sinciciais também foi menor em relação ao controle, diferindo significativamente (Tab. 5). O teste de Tunel mostrou atividade apoptótica apenas nas placentas com 14 dias de desenvolvimento de ambos os grupos, sendo mais intensa no grupo tratado (Fig. 2A-H). Não foram observados indícios de malformação na cabeça, no tronco e nos membros dos neonatos. No entanto, houve uma redução significativa no número e no peso dos neonatos no grupo tratado com o enrofloxacina em relação ao controle, porém sem afetar o seu comprimento (Tab. 6).

Tabela 3. *Médias e desvio-padrão da área $\left(\mu \mathrm{m}^{2}\right)$ total do disco placentário aos 14 e 20 dias de prenhez das ratas dos grupos experimentais

\begin{tabular}{cccc}
\hline Dias & GI & GII & F $^{P}$ \\
\hline 14 & $3754,98 \pm 18,65 \mathrm{a}$ & $2487,66 \pm 12,43 \mathrm{~b}$ & $5,270^{0,0354}$ \\
20 & $5845,50 \pm 17,71 \mathrm{a}$ & $3143,09 \pm 14,05 \mathrm{~b}$ & $1,584^{0,0160}$
\end{tabular}

*Médias seguidas pela mesma letra não diferem significativamente entre si pelo teste de Wilcoxon-Mann-Whitney $(\mathrm{p}<0,05)$.

Tabela 4. *Médias e desvio-padrão dos elementos constituintes da região do labirinto do disco placentário, com 14 dias de desenvolvimento nos grupos experimentais

\begin{tabular}{ccccc}
\hline DIAS & & GI & GII & $F^{P}$ \\
\hline \multirow{2}{*}{14} & VM & $16,55 \pm 1,21 \mathrm{a}$ & $14,98 \pm 1,09 \mathrm{~b}$ & $3,363^{0,0205}$ \\
& VF & $13,22 \pm 1,73 \mathrm{a}$ & $11,00 \pm 0,80 \mathrm{~b}$ & $2,546^{0,0044}$ \\
& TS & $63,11 \pm 1,32 \mathrm{a}$ & $58,67 \pm 2,19 \mathrm{~b}$ & $5,112^{0,0441}$ \\
\hline \multirow{2}{*}{20} & VM & $22,86 \pm 0,37 \mathrm{a}$ & $18,94 \pm 1,09 \mathrm{~b}$ & $1,854^{0,0276}$ \\
& VF & $18,99 \pm 1,11 \mathrm{a}$ & $16,45 \pm 0,65 \mathrm{~b}$ & $2,111^{0,0464}$ \\
& TS & $70,73 \pm 1,06 \mathrm{a}$ & $67,18 \pm 2,35 \mathrm{~b}$ & $4,197^{0,0342}$
\end{tabular}

*Médias seguidas pela mesma letra não diferem significativamente entre si pelo teste de Wilcoxon-Mann-Whitney $(\mathrm{P}<0,05)$.

$\mathrm{VM}=$ vasos maternos $; \mathrm{VF}=$ vasos fetais e $\mathrm{TS}=$ trofoblasto sincicial. 
Tabela 5. *Médias e desvio-padrão dos elementos constituintes da região do trofospongio e das células trofoblásticas gigantes do disco placentário, com 14 dias de desenvolvimento, nos grupos experimentais

\begin{tabular}{ccccc}
\hline Dias & & GI & GII & F $^{\mathrm{P}}$ \\
\hline \multirow{2}{*}{14} & CT & $45,22 \pm 1,56 \mathrm{a}$ & $41,02 \pm 1,92 \mathrm{~b}$ & $1,845^{0,0122}$ \\
& TS & $8,44 \pm 0,83 \mathrm{a}$ & $6,27 \pm 0,47 \mathrm{~b}$ & $4,132^{0,0498}$ \\
& CTG & $68,85 \pm 1,84 \mathrm{a}$ & $63,68 \pm 2,77 \mathrm{~b}$ & $5,511^{0,0483}$ \\
\hline \multirow{2}{*}{20} & CT & $57,19 \pm 1,94 \mathrm{a}$ & $54,99 \pm 1,26 \mathrm{~b}$ & $2,303^{0,0295}$ \\
& TS & $16,69 \pm 1,53 \mathrm{a}$ & $14,01 \pm 1,08 \mathrm{~b}$ & $1,756^{0,0374}$ \\
& CTG & $75,39 \pm 1,64 \mathrm{a}$ & $72,15 \pm 2,33 \mathrm{~b}$ & $4,197^{0,0243}$ \\
\hline
\end{tabular}

*Médias seguidas pela mesma letra não diferem significativamente entre si pelo teste de Wilcoxon-Mann-Whitney $(\mathrm{P}<0,05)$.

$\mathrm{CT}=$ células trofoblásticas; $\mathrm{TS}=$ trofoblasto sincicial e $\mathrm{CTG}=$ células trofoblásticas gigantes.

Tabela 6. *Médias e desvio-padrão do peso $(\mathrm{g})$ e comprimento $(\mathrm{cm})$ dos filhotes com um dia de nascido dos grupos experimentais

\begin{tabular}{cccc}
\hline & GI & GII & $\mathrm{F}^{\mathrm{P}}$ \\
\hline Número & $12,00 \pm 1,58 \mathrm{a}$ & $9,20 \pm 1,78 \mathrm{~b}$ & $1,543^{0,0211}$ \\
Peso & $7,65 \pm 0,28 \mathrm{a}$ & $6,54 \pm 0,38 \mathrm{~b}$ & $4,113^{0,0015}$ \\
Comprimento & $6,70 \pm 0,24 \mathrm{a}$ & $6,56 \pm 0,26 \mathrm{a}$ & $1,001^{0,0096}$ \\
\hline
\end{tabular}

*Médias seguidas pela mesma letra não diferem significativamente entre si pelo teste de Wilcoxon-Mann-Whitney $(\mathrm{P}<0,05)$.

\section{DISCUSSÃO}

A implantação embrionária é o processo pelo qual o embrião, sob a forma de blastocisto, adquire uma posição estável no endométrio, de modo a tornar possível a manutenção de um eficiente sistema de trocas metabólicas e de informações entre o organismo materno e o embrionário. $\mathrm{O}$ uso de medicamentos na gestação merece especial atenção pelos riscos potenciais ao feto em desenvolvimento (RedHorse, 2004). Os resultados mostraram uma diminuição significativa no número de sítios de implantação nas ratas tratadas com enrofloxacina na dosagem de $5 \mathrm{mg} / \mathrm{kg}$, durante os sete primeiros dias de gestação, em relação ao grupo controle. No período da implantação, o blastocisto parece ser muito sensível a ações de substâncias químicas, o que resulta, muitas vezes, na morte dele (Kim et al., 2003). Isto sugere que a enrofloxacina pode ter tido uma ação embriocida e, como consequência, reduzido, de forma considerável, o número de neonatos, semelhantemente ao que ocorreu às fluoroquinolonas DW -16 e norfloxacina, que, quando administradas em altas dosagens $(500 \mathrm{mg} / \mathrm{kg}$ e $400 \mathrm{mg} / \mathrm{kg}$, respectivamente) em ratas (Kim et al., 2000) e macacas (Corrado et $a l .$, 1987), produziram perda de embriões.
A redução do peso, da área total e dos constituintes das placentas com 14 e 20 dias de desenvolvimento, observada nas fêmeas do grupo tratado, pode estar relacionada ao fato de que as fluoroquinolonas podem produzir um retardo no crescimento uterino ou, ainda, promover a diminuição na ingestão de alimentos (Kim et al., 2000). De uma forma ou de outra, mesmo não havendo malformação, houve um reflexo no peso da prole em decorrência dessas alterações, pois nos mamíferos a implantação é considerada o ponto crítico da gravidez, e o sucesso da gestação requer o desenvolvimento normal de uma interação sincronizada entre o endométrio e o blastocisto (Cross et al., 1994), a qual permite o desenvolvimento fetal normal (Weissbluth e Bakos, 1992).

O aumento do índice apoptótico das células da placenta, como o trofoblasto, pode afetar sua função placentária e, consequentemente, resultar em gestações complicadas (Smith et al., 1999), o que, em humanos, por exemplo, leva ao retardo do crescimento uterino e ao aumento de aborto espontâneo no primeiro trimestre de gravidez (Kokawa et al., 1998). Em camundongos e ratos, várias substâncias tóxicas podem induzir a apoptose placentária excessiva bem como prejudicar o crescimento fetal (Thota et al., 2005), como, por exemplo, as fluoroquinolonas, 
as quais agem inibindo as enzimas que são fundamentais para replicação e transcrição do DNA e, assim, promovem a morte celular (Bhanot et al., 2001). Além disso, análises imuno-histoquímicas em placenta de vacas com quatro, seis e nove meses de gestação mostraram que a apoptose estava presente em todos os períodos gestacionais estudados, sendo maior no grupo com nove meses de gestação, o que indica que a via apoptótica é mais ativa durante a maturação placentária (Meça et al., 2011). Isso poderia explicar a atividade apoptótica mais intensa observada apenas nas placentas com 14 dias de desenvolvimento, período de maturação placentária nos roedores (Caluwaerts et al., 2005), porém o tratamento com a enrofloxacina parece estimular essa atividade, pois foi mais intensa no grupo tratado.

A análise histoquímica revelou que não houve alterações significativas no teor de fibras colágenas, elásticas e reticulares. Esse resultado pode estar associado à dosagem utilizada neste experimento, visto que, em doses elevadas (superiores a $30 \mathrm{mg} / \mathrm{kg}$ ), algumas fluoroquinolonas causam degradação dos constituintes fibrosos da matriz extracelular dos tendões, devido à quelação do magnésio que alteraria as proteínas dos tendões, principalmente o colágeno, a elastina e a fibronectina (Stahlmann, 2003).

\section{CONCLUSÃO}

A enrofloxacina na dosagem de $5 \mathrm{mg} / \mathrm{kg}$ durante prenhez em ratas interfere número de embriões implantados e no desenvolvimento placentário. Isto sugere cautela na administração da enrofloxacina durante a gestação, pois a exposição contínua a esse antibiótico pode ter reflexos na redução do número e no peso da prole.

\section{AGRADECIMENTOS}

Ao Conselho Nacional de Desenvolvimento Científico e Tecnológico (CNPq), pela concessão da bolsa.

\section{REFERÊNCIAS}

ABD-ALLAH, A.R.A.; GANNAHAM， B.B.; HAMADA, A.M. The impact of ofloxacin on rat testicular DNA: application of image analysis. Pharmacol. Res., v.42, p.145-150, 2000.

ANDRADE, A. Segurança em biotérios. In: Andrade, A.; Pinto, S.C.; Oliveira, R.S. (orgs.). Animais de laboratório: criação e experimentação. Rio de Janeiro: Editora Fiocruz, 2002. p.381-387.

ARAL, F.; KARACAL, F.; BABA, F. The effect of enrofloxacin on sperm quality in male mice. Res. Vet. Sci., v.84, p.95-99, 2008.

BHANOT, S.K.; SINGH, M.; CHATTERJEE, N.R. The chemical and biological aspects of fluoroquinolones: reality and dreams. Curr. Pharm. Des., v.7, p.311-335, 2001.

CALUWAERTS, S.; VERCRUYSSE, L.; LUYTEN, C. et al. Endovascular trophoblast invasion and associated structural changes in uterine spiral arteries of the pregnant rat. Placenta, v.26, p.574-584, 2005.

CORRADO, M.L.; STRUBLE, W.E.; PETER, C. et al. Norfloxacin: review of safety studies. Am. J. Med., v.82, p.22-26, 1987.

CROSS, J.C.; WERB, Z.; FISHER, S.J. Implantation and the placenta: key pieces of the development puzzle. Science. v.266, p.15081518, 1994.

ELMAS, M.; TIRAS, B.; KAYA, S. et al. Pharmacokinetics of enrofloxacin after intravenous and intramuscular administration in angora goats were studied. Can. J. Vet. Res., v.65, p.64-67, 2001.

ELMAS, M.; ÜNEY, K.; YAZAR, E. et al. Pharmacokinetics of enrofloxacin following intravenous and intramuscular administration in Angora rabbits. Res. Vet. Sci., v.82, p.242-245, 2007.

GURBAY, A.; GONTHIER, B.; SIGNORINI ALLIBE, N. Ciprofloxacin induced DNA damage in primary culture of rat astrocytes and protection by vitamin E. Neurotoxicology, v.27, p.6-10, 2006. 
KATAYAMA, K.I.; UENO, M.; TAKAI, H. et al. Ethylnitrosourea induces apoptosis and growth arrest in the trophoblastic cells of rat placenta. Biol. Reprod., v.67, p.431-435, 2002.

KIM, J.C.; BAE, C.S.; KIM, S.H. et al. Transplacental pharmacokinetics of the new fluoroquinolone DW-116 in pregnant rats. Toxicol. Letter., v.142, p.103-109, 2003.

KIM, J.C.; YUN, H.I.; SHIN, H.C. et al. Embryo lethality and teratogenicity of a new fluoroquinolone antibacterial $\mathrm{dw}-116$ in rats. Arch. Toxicol., v.74, p. 120-124, 2000.

KOKAWA, K.; SHIKONE, T.; NAKANO, R. Apoptosis in human chorionic villi and decidua during normal embryonic development and spontaneous abortion in the first trimester. Placenta, v.19, p.21-26, 1998.

LEMUS, J.A.; BLANCO, G.; ARROYO, B. et al. Fatal embryo chondral damage associated with fluoroquinolones in eggs of threatened avian scavengers. Environ. Pollut., v.157, p.2421-2427, 2009.

LEMUS, J.A.; BLANCO, G.; GRANDE, J. et al. Antibiotics threaten wildlife: circulating quinolone residues and disease in avian scavengers. Plos. One, v.3, p.144-1449, 2008.

LIM, S.; HOSSAIN, M.A, PARK, J, et al. . The effects of enrofloxacin on canine tendon cells and chondrocytes proliferation in vitro. Vet. Res. Commun., v.32, p.243-253, 2008.

MARÍN, P.; ESCUDERO, E.; FERNÁNDEZVARÓN, E. Pharmacokinetics and milk penetration of orbifloxacin after intravenous, subcutaneous, and intramuscular administration to lactating goats. J. Dairy. Sci., v.90, p.42194225, 2007.

MAYHEW, T.M. Stereology and the placenta: where's the point? - A review. Placenta v.27, p.S17-S25, 2006.

MEÇA, K.K.O.L.; PUERTO, H.L.D.; RODRIGUES, L.V. et al. Apoptose na maturação placentária de vacas em diferentes estágios de gestação: evidenciação imunohistoquímica e bioquímica. Pesq. Vet. Bras., v.31, p.718-722, 2011.

MINTA, M.; IWONA, W.; JAN, Z. Inhibition of cell differentiation by quinolones in micromass cultures of rat embryonic limb bud and midbrain cells. Toxicol. in Vitro, v.19, p.915-919, 2005.

OTERO, J.L.; MESTORINO, N.; ERRECALDE, J.O. Enrofloxacina: una fluorquinolona de uso exclusivo em veterinaria. Parte II: farmacocinetica y tocicidad. Anal. Vet., v.21, p.42-49, 2001.

OWENS, R.C.J.R.; AMBROSE, P.G. Antimicrocrobial safety: focus on fluoroquinolones: focus on fluoroquinolones. Clin. Infect. Dis., v.15, p.144-157, 2005.

RED-HORSE, K.; ZHOU, Y.; GENBACEV, O. et al. Trophoblast differentiation during embryo implantation and formation of the maternal-fetal interface. J. Clin. Invest., v.114, p.744-754, 2004.

SHEHATA, H.A.; NELSON-PIERCY, C. Drugs to avoid. Best Pract. Res. Clin. Obstet. Gynaecol., v.15, p.971-986, 2001.

SMITH, S.C.; BAKER, P.N.; SYMONDS, E.M. Placental apoptosis in normal human pregnancy. Am. J. Obstet. Gynecol., v.177, p.57-65, 1999.

STAHLMANN, R. Clinical toxicological aspects of fluoroquinolones. Toxicol. Lett., v.27, p.269277, 2002.

STAHLMANN, R. Children as a special population at risk - quinolones as an example for xenobiotics exhibiting skeletal toxicity. Arch. Toxicol., v.77, p.7-11, 2003.

THOTA, C.S.; REED, L.C.; YALLAMPALLI, C. Effects of parathyroid hormone like hormone (PTHLH) antagonist, PTHLH(7-34), on fetoplacental development and growth during midgestation in rats. Biol. Reprod., v.73, p.11911198, 2005.

VAN DER LINDEN, P.D.; STURKENBOOM, M.C.; HERINGS, R.M. et al. Increased risk of Achilles tendon rupture with quinolone antibacterial use, especially in elderly patients taking oral corticosteroids. Arch. Intern. Med., v.163, p.1801-1807, 2003.

WEISSBLUTH, M.L.; BAKOS, L. Terapêutica dermatológica sistêmica e tópica na gravidez e lactação: o que pode ser usado? An. Bras. Dermatol., v.67, p.211-216, 1992. 